\section{A neural-holographic model of sensory and memorial oblique effects}

\section{ETHEL MATIN and JAMES THOMS \\ C. W. Post Center, Long Island University \\ Greenvale, New York}

This note's purpose is to outline a model of the oblique effect. It was inspired by findings about a memorial manifestation of the oblique phenomenon that appeared in a study of memory for the orientation of a 3.8-cpd sinusoidal grating at two reference orientations, 90 and $45 \mathrm{deg}$ (Thoms, 1981). The results of the study suggested an exponential decay process that could be related, in some rather surprising ways, to current thinking about the corresponding sensory oblique effect.

In Thoms's study, measurements were made with a delayed comparison procedure as follows: On each trial, the grating was flashed twice for $200 \mathrm{msec}$ with an interstimulus interval ranging from .35 to $2.8 \mathrm{sec}$. Observers were required to report whether the grating's orientation was the same or different on the two presentations. For each of the four subjects studied, and for both the vertical and the oblique gratings, discriminability decreased with increase in interstimulus interval. But the results for the 90 -deg reference orientation and the 45-deg orientation differed in two important ways: First, orientation discrimination was poorer at $45 \mathrm{deg}$ than at $90 \mathrm{deg}$ for all interstimulus intervals. Second, the rate of decay of discriminability was slower at $45 \mathrm{deg}$ than at $90 \mathrm{deg}$. The latter finding, which is in line with the results of another recent study showing longer visible persistence at oblique meridians relative to persistence at the main meridians (Bowling \& Lovegrove, 1981), provided the hint that led to our theoretical conceptions.

The neural model of the oblique effect appears in the first section of this paper after the presentation of a brief outline of background information needed to develop our arguments. The second section relates the model from the first section to ideas about holographic information storage with suggestions about a physiological realization of a hologram.

\section{Neural Modeling of the Oblique Effect}

The oblique effect, which we define as sensory, perceptual, or cognitive performance that depends on stimulus orientation, was first formally examined

This report was supported by RO1EY02951 from the National Eye Institute and by an award from the Research Committee of the C. W. Post Center, LIU. Requests for reprints should be sent to Ethel Matin, Department of Psychology, C. W. Post Center, Long Island University, Greenvale, NY 11548. in a study published near the turn of the century by Jastrow (1893). It continued to receive sporadic experimental attention in the psychological literature for the next $\mathbf{7 5}$ years. This relatively fallow period was followed by a burst of intensified effort, stimulated and focused by Hubel and Wiesel's discovery of orientation-tuned cortical cells in the mammalian visual system (Hubel \& Wiesel, 1962, 1965, 1968). Early in the modern period of research, possible optical causes of the phenomenon were ruled out by studies showing that it persisted when the optics of the eye were bypassed with a stimulus arrangement for generating interference fringes on the retina (Campbell, Kulikowski, \& Levinson, 1966; Mitchell, Freeman, \& Westheimer, 1967). Peripheral neural factors were also eliminated as causes when the effect appeared in the response evoked at the cortex but not in the electroretinogram (Maffei \& Campbell, 1970). Around the same time, two models were proposed to account for the various facts of orientation discrimination, including the oblique effect, in terms of the newly discovered tilt detectors. One postulated sharper tuning for cortical cells with preferred orientations at the main meridians relative to cells with oblique preferred orientations (Andrews, 1967). The other was based on the possibility that the number of orientation-tuned cells was greater at the main meridians than at the obliques (Bouma \& Andriessen, 1968). A third possibility was added to the others when Rose and Blakemore (1974) included lower thresholds for cortical cells at the main meridians among the suggested neural causes of various psychophysical manifestations of meridional anistropy.

When the various possible neural substrates for the oblique effect were first proposed, little electrophysiological evidence was available for choosing among them. Since then, however, most of the data that have appeared in the literature showed no significant differences in orientation tuning among cells with different preferred orientations (e.g., DeValois, Yund, \& Hepler, 1982; Mansfield, 1975). Nor does there appear to be any significant difference in threshold (Rose \& Blakemore, 1974). However, several groups of investigators have found that the number of cells with preferred orientation at the main meridians is greater than the number tuned to the obliques for cells with foveal receptive fields (DeValois et al., 1982; Heggelund \& Albus, 1978; Leventhal \& Hirsch, 1980; Mansfield, 1975; Pettigrew, Nikara, \& Bishop, 1968). Another pair of investigators (Orban \& Kennedy, 1981) reported that a meridional anisotropy in number of neurons exists but only in area 17 and only in cells they classified in the " $S$ " group, which appears to be a subset of the simple cells. Related psychophysical evidence implicating the sus- 
tained visual channels has been found in several studies. This evidence appears, for example, in the experiments of Berkley, Kitterle, and Watkins (1975), who found a diminishing of the oblique effect with retinal eccentricity, and in those of Camisa, Blake, and Lema (1977), who reported that it occurs only with high spatial and low temporal frequencies.

On the strength of the evidence cited in the preceding paragraph, several writers have supported the suggestion that various sensory manifestations of the oblique effect could be related to meridional differences in the number of orientation-tuned cells (e.g., DeValois et al., 1982; Mansfield, 1975; Stone, Dreher, \& Leventhal, 1979). However, they have not attempted to explain how a larger number of cells could create superior performance. We will make a specific suggestion on that issue in the next section. For the moment, however, we simply join the developing consensus of opinion on the matter and incorporate it in the following theoretical suggestions.

(1) The number of responding orientation-tuned cells in an appropriately chosen subset of such cells provides the neural basis of various sensory manifestations of the oblique effect, such as meridional differences in contrast sensitivity, acuity, and orientation sensitivity.

(2) From the fact that memorial orientation sensitivity for all interstimulus intervals is greater at $90 \mathrm{deg}$ than at $45 \mathrm{deg}$ (Thoms, 1981), as has been found for the corresponding sensory effect (Bouma \& Andriessen, 1968; Matin \& Drivas, 1979), it seems reasonable to suggest that storage of information about the 90-deg orientation, like its processing during ongoing stimulation, also involves a larger number of orientation-tuned cells relative to the number involved for the 45-deg stimulus.

(3) Finally, if we assume that the number of responding neurons in the relevant set decreases with time after stimulus offset at a rate that depends at any moment on the number of firing cells, we could account for the slower memorial decay for the 45-deg stimulus relative to that at $90 \mathrm{deg}$.

The possibility that memorial strength and degree of sensitivity to slant are both coded in terms of the total number of orientation-tuned cells does not of itself require an identity between the set of storage cells and the set of cells involved in sensory processing. In fact, three logical possibilities exist: identical sets, disjoint sets, and sets that share some neurons. The first of these seems unlikely-simply because of the phenomenal differences between a memory and a sensation and because of their different functional roles. The second (disjoint sets) is tacitly understood in most approaches to memory and certainly has a lot to recommend it. Nonetheless, we confess to being particularly intrigued by the third possibility-specifically, by the idea that the memory set could be a subset of the sensory set. Such an arrangement would have several attractive features, including a very useful economy in the deployment of available neurons.

\section{Holographic Storage}

Although it may not at first be obvious, our suggestions about sensitivity and amount of stored information in terms of the number of responding neurons can be related in a rather concrete way to ideas about holographic methods of information storage in the brain. (See Pribram, Nuwer, \& Baron, 1974, and Weisstein \& Harris, 1980, for discussions of holography and memory.) To show how this can be done, we call the reader's attention to the following property of a holographic image: overall structure of the object is retained while total amount of information (detail and resolving power) is decreased if pieces of the hologram are replaced by opacities. Then, to establish the linkage to the previously discussed physiological concepts, we treat the neurons that cease to respond during memorial decay as the analogue of the lost (opaque) pieces of the hologram. With an increase in the opaque areas (in essence, with a decrease in the effective size of the hologram), there occurs a decrease in the amount of information-specifically, in the case we are considering, diminished information about slant.

These ideas about a physiological realization of a hologram in terms of a set of responding neurons are compatible both with models specifying disjoint sets for storage and for processing and with models treating the memory set as a subset of the sensory set. In the case of the former, the processing of data and its storage would be accomplished in separate holographiclike mechanisms (i.e., mechanisms that code amount of information in terms of the total effective size of the coding element). For the second type of model, one such holographic mechanism could be used for both processing and for storage. The difference between a sensation and an image in short-term memory could then simply be a difference in the number of responding neurons: The full set involved in sensation gradually decreases in size; after some threshold amount of decrease, the persisting sensation becomes an image in short-term memory.

\section{REFERENCES}

Andrews, D. (1967). Perception of contour orientation in the central fovea. Part I: Short lines. Vision Research, 7, 979-997.

Benkley, M., Kitterle, F., \& Watkins, D. (1975). Grating visibility as a function of orientation and retinal eccentricity. Vision Research, 15, 239-244.

Bouma, H., \& Andriessen, J. (1968). Perceived orientation of isolated line segments. Vision Research, 8, 493-507.

Bowling, A., \& Lovegrove, W. (1981). Two components to visible persistence: Effects of orientation and contrast. Vision Research, 21, 1241-1251.

Camisa, J., Blake, R., \& Lema, S. (1977). The effects of temporal modulation on the oblique effect in humans. Perception, 6, 165-171. 
Campbell, F., Kulikowski, J., \& Levinson, J. (1966). The effects of orientation on the visual resolution of gratings. Journal of Physiology, 187, 427-436.

DeValois, R., Yund, E., \& Heplet, N. (1982). The orientation and direction selectivity of cells in macaque visual cortex. Vision Research, 22, 531-544.

Heggelund, P., \& Albus, K. (1978). Response variability and orientation discrimination of single cells in striate cortex of cat. Experimental Brain Research, 32, 197-211.

Hubel, D., \& Wiesel, T. (1962). Receptive fields, binocular interaction and functional architecture in the cat visual cortex. Journal of Physiology, 160, 106-154.

HuBel, D., \& WIESEL, T. (1965). Receptive fields and functional architecture in two non-striate visual areas (18 and 19) of the cat. Journal of Neurophysiology, 28, 229-289.

Hubel, D., \& Wiesel, T. (1968). Receptive fields and functional architecture of monkey striate cortex. Journal of Physiology, 195, 215-243.

JAsTROW. J. (1893). On the judgment of angles and positions of lines. American Journal of Psychology, 5, 214-248.

Leventhal, A., \& Hinsch, H. (1980). Receptive field properties of different classes of neurons in visual cortex of normal and dark reared cats. Journal of Neurophysiology, 43, 1111-1132.

MAFFE I, L, \& CAMPBELL, F. (1970). Neurophysiological localization of vertical and horizontal visual coordination in man. Science, 167, 386-387.

MANSFIELd, R. (1975). Neural basis of orientation perception in primate vision. Science, 186, 1133-1135.

Matin, E., \& Drivas, A. (1979). Acuity for orientation measured with a signal recognition task and signal detection methods. Perception \& Psychophysics, 25, 161-168.
Mitchell, D., Freeman, R., \& Westheimer, G. (1967). Effect of orientation on the modulation sensitivity for interference fringes on the retina. Journal of the Optical Society of America, 57, 246-249.

Orban, G., \& Kennedy, H. (1981). The influence of eccentricity on receptive field types and orientation selectivity in areas 17 and 18 of the cat. Brain Research, 208, 203-208.

Pettighew, J., Nikara, T., \& Bishop, P. (1968). Responses to moving slits in cat striate cortex. Experimental Brain Research, 6, 373-390.

Pribram, K., Nuwer, M., \& Baron, R. (1974). The holographic hypothesis of memory structure in brain function and perception. In R. Atkinson, R. Luce, \& P. Suppes, (Eds.), Contemporary developments in mathematical psychology (Vol. II). San Francisco: Freeman.

Rose, D., \& Blakemore, C. (1974). An analysis of orientation selectivity in the cat's visual cortex. Experimental Brain Research, 20, 1-17.

Stone, J., Dreher, B., \& Leventhal, A. (1979). Hierarchical and parallel mechanisms in the organization of visual cortex. Brain Research Reviews, 1, 345-394.

Tномs, J. (1981). The oblique effect in short term memory. Unpublished master's thesis. C. W. Post Center, Long Island University.

We Isste in, N., \& HaRRis, C. (1980). Masking and unmasking of distributed representations in the visual system. In C. Harris (Ed.), Visual coding and adaptability. Hillsdale, NJ: Erlbaum.

(Manuscript received April 19, 1984; accepted for publication April 19, 1984. ) 